\title{
Systemic Corticosteroids in Critically III Patients With COVID-19
}

WHO Rapid Evidence Appraisal for COVID-19 Therapies (REACT) Working Group, Sterne JAC, Murthy S, Diaz JV, et al. Association between administration of systemic corticosteroids and mortality among critically ill patients with COVID-19: a meta-analysis. JAMA. 2020 Sep 2. doi:10.1001/ jama.2020.17023.

\section{Study Overview}

Objective. To assess the association between administration of systemic corticosteroids, compared with usual care or placebo, and 28-day all-cause mortality in critically ill patients with coronavirus disease 2019 (COVID-19).

Design. Prospective meta-analysis with data from 7 randomized clinical trials conducted in 12 countries.

Setting and participants. This prospective meta-analysis included randomized clinical trials conducted between February 26, 2020, and June 9, 2020, that examined the clinical efficacy of administration of corticosteroids in hospitalized COVID-19 patients who were critically ill. Trials were systematically identified from ClinicalTrials.gov, the Chinese Clinical Trial Registry, and the EU Clinical Trials Register, using the search terms COVID-19, corticosteroids, and steroids. Additional trials were identified by experts from the WHO Rapid Evidence Appraisal for COVID-19 Therapies (REACT) Working Group. Senior investigators of these identified trials were asked to participate in weekly calls to develop a protocol for the prospective meta-analysis. ${ }^{1}$ Subsequently, trials that had randomly assigned critically ill patients to receive corticosteroids versus usual care or placebo were invited to participate in this meta-analysis. Data were pooled from patients recruited to the participating trials through June 9, 2020, and aggregated in overall and in predefined subgroups.

Main outcome measures. The primary outcome was allcause mortality up to 30 days after randomization. Because 5 of the included trials reported mortality at 28 days after randomization, the primary outcome was reported as 28-day all-cause mortality. The secondary outcome was serious adverse events (SAEs). The authors also gathered data on the demographic and clinical characteristics of patients, the number of patients lost to follow-up, and outcomes according to intervention group, overall, and in subgroups (ie, patients receiving invasive mechanical ventilation or vasoactive medication; age $\leq 60$ years or $>60$ years [the median across trials]; sex [male or female]; and the duration patients were symptomatic $[\leq 7$ days or $>7$ days]). For each trial, the risk of bias was assessed independently by 4 investigators using the Cochrane Risk of Bias Assessment Tool for the overall effects of corticosteroids on mortality and SAEs

\section{Outcomes Research in Review SECTION EDITORS}

Katrina F. Mateo, PhD, MPH

CUNY School of Public Health New York, NY

TAISHI HIRAI, MD

University of Missouri

Columbia, MO
DANIEL ISAAC, DO, MS

Michigan State University

East Lansing, MI

WILLIAM HUNG, MD, MPH

Icahn School of Medicine at Mount Sinai

New York, NY
FRED Ko, MD, MS

Icahn School of Medicine at Mount Sinai New York, NY

GoRdON NGAI, MD, MPH

Icahn School of Medicine at Mount Sinai New York, NY 


\section{Outcomes Research in Review}

and the effect of assignment and allocated interventions. Inconsistency between trial results was evaluated using the $R^{2}$ statistic. The trials were classified according to the corticosteroids used in the intervention group and the dose administered using a priori-defined cutoffs (15 $\mathrm{mg} /$ day of dexamethasone, $400 \mathrm{mg} /$ day of hydrocortisone, and $1 \mathrm{mg} / \mathrm{kg} /$ day of methylprednisolone). The primary analysis utilized was an inverse variance-weighted fixed-effect meta-analysis of odds ratios (ORs) for overall mortality. Random-effects meta-analyses with PauleMandel estimate of heterogeneity were also performed.

Main results. Seven trials (DEXA-COVID 19, CoDEX, RECOVERY, CAPE COVID, COVID STEROID, REMAPCAP, and Steroids-SARI) were included in the final meta-analysis. The enrolled patients were from Australia, Brazil, Canada, China, Denmark, France, Ireland, the Netherlands, New Zealand, Spain, the United Kingdom, and the United States. The date of final follow-up was July 6, 2020. The corticosteroids groups included dexamethasone at low (6 mg/day orally or intravenously [IV]) and high (20 mg/day IV) doses; low-dose hydrocortisone (200 mg/day IV or 50 mg every 6 hr IV); and high-dose methylprednisolone (40 mg every $12 \mathrm{hr} \mathrm{IV).} \mathrm{In} \mathrm{total,} 1703$ patients were randomized, with 678 assigned to the corticosteroids group and 1025 to the usual-care or placebo group. The median age of patients was 60 years (interquartile range, 52-68 years), and 29\% were women. The larger number of patients in the usual-care/placebo group was a result of the 1:2 randomization (corticosteroids versus usual care or placebo) in the RECOVERY trial, which contributed $59.1 \%$ of patients included in this prospective meta-analysis. The majority of patients were receiving invasive mechanical ventilation at randomization (1559 patients). The administration of adjunctive treatments, such as azithromycin or antiviral agents, varied among the trials. The risk of bias was determined as low for 6 of the 7 mortality results.

A total of 222 of 678 patients in the corticosteroids group died, and 425 of 1025 patients in the usual care or placebo group died. The summary OR was 0.66 (95\% confidence interval [Cl], 0.53-0.82; $P<0.001$ ) based on a fixed-effect meta-analysis, and 0.70 (95\% Cl, 0.48-1.01; $P=0.053)$ based on the random-effects meta-analysis, for 28-day all-cause mortality comparing all corticosteroids with usual care or placebo. There was little inconsistency between trial results $\left(I^{2}=15.6 \% ; P=0.31\right)$. The fixed-effect summary OR for the association with 28-day all-cause mortality was $0.64(95 \% \mathrm{Cl}, 0.50-0.82 ; P<$ 0.001) for dexamethasone compared with usual care or placebo (3 trials, 1282 patients, and 527 deaths); the OR was $0.69(95 \% \mathrm{Cl}, 0.43-1.12 ; P=0.13)$ for hydrocortisone ( 3 trials, 374 patients, and 94 deaths); and the OR was 0.91 (95\% Cl, 0.29-2.87; $P=0.87$ ) for methylprednisolone (1 trial, 47 patients, and 26 deaths). Moreover, in trials that administered low-dose corticosteroids, the overall fixed-effect OR for 28-day all-cause mortality was 0.61 (95\% Cl, 0.48-0.78; $P<0.001)$. In the subgroup analysis, the overall fixed-effect OR was $0.69(95 \% \mathrm{Cl}, 0.55-0.86)$ in patients who were receiving invasive mechanical ventilation at randomization, and the OR was $0.41(95 \% \mathrm{Cl}$, 0.19-0.88) in patients who were not receiving invasive mechanical ventilation at randomization.

Six trials (all except the RECOVERY trial) reported SAEs, with 64 events occurring among 354 patients assigned to the corticosteroids group and 80 SAEs occurring among 342 patients assigned to the usual-care or placebo group. There was no suggestion that the risk of SAEs was higher in patients who were administered corticosteroids.

Conclusion. The administration of systemic corticosteroids was associated with a lower 28-day all-cause mortality in critically ill patients with COVID-19 compared to those who received usual care or placebo.

\section{Commentary}

Corticosteroids are anti-inflammatory and vasoconstrictive medications that have long been used in intensive care units for the treatment of acute respiratory distress syndrome and septic shock. However, the therapeutic role of corticosteroids for treating severe acute respiratory syndrome coronavirus 2 (SARS-CoV-2) infection was uncertain at the outset of the COVID-19 pandemic due to concerns that this class of medications may cause an impaired immune response in the setting of a lifethreatening SARS-CoV-2 infection. Evidence supporting this notion included prior studies showing that corticosteroid therapy was associated with delayed viral clearance 
of Middle East respiratory syndrome or a higher viral load of SARS-CoV. ${ }^{2,3}$ The uncertainty surrounding the therapeutic use of corticosteroids in treating COVID-19 led to a simultaneous global effort to conduct randomized controlled trials to urgently examine this important clinical question. The open-label Randomized Evaluation of COVID-19 Therapy (RECOVERY) trial, conducted in the UK, was the first large-scale randomized clinical trial that reported the clinical benefit of corticosteroids in treating patients hospitalized with COVID-19. Specifically, it showed that low-dose dexamethasone (6 mg/day) administered orally or IV for up to 10 days resulted in a $2.8 \%$ absolute reduction in 28-day mortality, with the greatest benefit, an absolute risk reduction of $12.1 \%$, conferred to patients who were receiving invasive mechanical ventilation at the time of randomization. ${ }^{4}$ In response to these findings, the National Institutes of Health COVID19 Treatment Guidelines Panel recommended the use of dexamethasone in patients with COVID-19 who are on mechanical ventilation or who require supplemental oxygen, and recommended against the use of dexamethasone for those not requiring supplemental oxygen. ${ }^{5}$

The meta-analysis discussed in this commentary, conducted by the WHO REACT Working Group, has replicated initial findings from the RECOVERY trial. This prospective meta-analysis pooled data from 7 randomized controlled trials of corticosteroid therapy in 1703 critically ill patients hospitalized with COVID-19. Similar to findings from the RECOVERY trial, corticosteroids were associated with lower all-cause mortality at 28 days after randomization, and this benefit was observed both in critically ill patients who were receiving mechanical ventilation or supplemental oxygen without mechanical ventilation. Interestingly, while the $\mathrm{OR}$ estimates were imprecise, the reduction in mortality rates was similar between patients who were administered dexamethasone and hydrocortisone, which may suggest a general drug class effect. In addition, the mortality benefit of corticosteroids appeared similar for those aged $\leq 60$ years and those aged $>60$ years, between female and male patients, and those who were symptomatic for $\leq 7$ days or $>7$ days before randomization. Moreover, the administration of corticosteroids did not appear to increase the risk of SAEs. While more data are needed, results from the RECOVERY trial and this prospective metaanalysis indicate that corticosteroids should be an essential pharmacologic treatment for COVID-19, and suggest its potential role as a standard of care for critically ill patients with COVID-19.

This study has several limitations. First, not all trials systematically identified participated in the meta-analysis. Second, long-term outcomes after hospital discharge were not captured, and thus the effect of corticosteroids on long-term mortality and other adverse outcomes, such as hospital readmission, remain unknown. Third, because children were excluded from study participation, the effect of corticosteroids on pediatric COVID19 patients is unknown. Fourth, the RECOVERY trial contributed more than $50 \%$ of patients in the current analysis, although there was little inconsistency in the effects of corticosteroids on mortality between individual trials. Last, the meta-analysis was unable to establish the optimal dose or duration of corticosteroid intervention in critically ill COVID-19 patients, or determine its efficacy in patients with mild-to-moderate COVID-19, all of which are key clinical questions that will need to be addressed with further clinical investigations.

The development of effective treatments for COVID-19 is critical to mitigating the devastating consequences of SARS-CoV-2 infection. Several recent COVID-19 clinical trials have shown promise in this endeavor. For instance, the Adaptive COVID-19 Treatment Trial (ACCT-1) found that intravenous remdesivir, as compared to placebo, significantly shortened time to recovery in adult patients hospitalized with COVID-19 who had evidence of lower respiratory tract infection. ${ }^{6}$ Moreover, there is some evidence to suggest that convalescent plasma and aerosol inhalation of IFN- $\kappa$ may have beneficial effects in treating COVID-19.,8 Thus, clinical trials designed to investigate combination therapy approaches including corticosteroids, remdesivir, convalescent plasma, and others are urgently needed to help identify interventions that most effectively treat COVID-19.

\section{Applications for Clinical Practice}

The use of corticosteroids in critically ill patients with COVID-19 reduces overall mortality. This treatment is inexpensive and available in most care settings, including 
low-resource regions, and provides hope for better outcomes in the COVID-19 pandemic.

Katerina Oikonomou, MD, PhD

General Hospital of Larissa, Larissa, Greece

Fred Ko, MD, MS

doi:10.12788/jcom.0023

\section{References}

1. Sterne JAC, Diaz J, Villar J, et al. Corticosteroid therapy for critically ill patients with COVID-19: A structured summary of a study protocol for a prospective meta-analysis of randomized trials. Trials. 2020;21:734.

2. Lee N, Allen Chan KC, Hui DS, et al. Effects of early corticosteroid treatment on plasma SARS-associated Coronavirus RNA concentrations in adult patients. J Clin Virol. 2004;31:304-309.
3. Arabi YM, Mandourah Y, Al-Hameed F, et al. Corticosteroid therapy for citically III patients with Middle East respiratory syndrome. Am J Respir Crit Care Med. 2018;197:757-767.

4. RECOVERY Collaborative Group, Horby P, Lim WS, et al. Dexamethasone in hospitalized patients with Covid-19 - preliminary report [published online ahead of print, $2020 \mathrm{Jul}$ 17]. N Engl J Med. 2020;NEJMoa2021436.

5. NIH COVID-19 Treatment Guidelines. National Institutes of Health. www.covid19treatmentguidelines.nih.gov/immune-based-therapy/ immunomodulators/corticosteroids/. Accessed September 11, 2020.

6. Beigel JH, Tomashek KM, Dodd LE, et al. Remdesivir for the treatment of Covid-19--preliminary report [published online ahead of print, 2020 May 22]. N Engl J Med. 2020;NEJMoa2007764.

7. Casadevall A, Joyner MJ, Pirofski LA. A randomized trial of convalescent plasma for covid-19-potentially hopeful signals. JAMA. 2020;324:455-457.

8. Fu W, Liu Y, Xia L, et al. A clinical pilot study on the safety and efficacy of aerosol inhalation treatment of IFN- $\kappa$ plus TFF2 in patients with moderate COVID-19. EClinicalMedicine. 2020;25:100478.

\section{Effect of a Smartphone App Plus an} Accelerometer on Physical Activity and Functional Recovery During Hospitalization After Orthopedic Surgery

van Dijk-Huisman HC, Weemaes ATR, Boymans TAEJ, et al. Smartphone app with an accelerometer enhances patients' physical activity following elective orthopedic surgery: a pilot study. Sensors (Basel). 2020;20:4317.

\section{Study Overview}

Objective. To investigate the potential of Hospital Fit (a smartphone application with an accelerometer) to enhance physical activity levels and functional recovery following orthopedic surgery.

Design. Nonrandomized, quasi-experimental pilot study.

Settings and participants. Patients scheduled for an elective total knee arthroplasty (TKA) or total hip arthroplasty (THA) at the orthopedic ward of Maastricht University Medical Center in Maastricht, the Netherlands, were invited to participate. Patients scheduled for surgery between January 2017 and December 2018 were recruited for the control group at a rate of 1 patient per week (due to a limited number of accelerometers available). After development of Hospital Fit was completed in December 2018 (and sufficient accelerators had become available), patients scheduled for surgery between February 2019 and May 2019 were recruited for the intervention group. The ratio of patients included in the control and intervention group was set at 2:1, respectively.

At preoperative physiotherapy screenings (scheduled 6 weeks before surgery), patients received verbal and written information about the study. Patients were eligible if they met the following inclusion criteria: receiving physiotherapy after elective TKA or THA; able to walk independently 2 weeks prior to surgery, as scored on the Functional Ambulation Categories (FAC > 3); were expected to be discharged to their own home; were aged 18 years and older; and had a sufficient understanding of the Dutch language. Exclusion criteria were: the presence of contraindications to walking or wearing an acceler- 


\section{Outcomes Research in Review}

ometer on the upper leg; admission to the intensive care unit; impaired cognition (delirium/dementia), as reported by the attending doctor; a life expectancy of less than 3 months; and previous participation in this study. Patients were contacted on the day of their surgery, and written informed consent was obtained prior to the initiation of any study activities.

Intervention. Once enrolled, all patients followed a standardized clinical care pathway for TKA or THA (see original article for additional details). Postoperative physiotherapy was administered to all participating patients, starting within 4 hours after surgery. The physiotherapy treatment was aimed at increasing physical activity levels and enhancing functional recovery. Control group patients only received physiotherapy (twice daily, 30 minutes per session) and had their physical activity levels monitored with an accelerometer, without receiving feedback, until functional recovery was achieved, as measured with the modified lowa Level of Assistance Scale (mILAS). Intervention group patients used Hospital Fit in addition to physiotherapy. Hospital Fit consists of a smartphone-based app, connected to a MOX activity monitor via Bluetooth (device contains a tri-axial accelerometer sensor in a small waterproof housing attached to the upper leg). Hospital Fit enables objective activity monitoring, provides patients and their physiotherapists insights and real-time feedback on the number of minutes spent standing and walking per day, and offers a tailored exercise program supported by videos aimed at stimulating self-management.

Measures. The primary outcome measure was the time spent physically active (total number of minutes standing and walking) per day until discharge. Physical activity was monitored 24 hours a day; days with $\geq 20$ hours of wear time were considered valid measurement days and were included in the analysis. After the last treatment session, the accelerometer was removed, and the raw tri-axial accelerometer data were uploaded and processed to classify minutes as "active" (standing and walking) or "sedentary" (lying and sitting). The secondary outcome measures were the achievement of functional recovery on postoperative day 1 (POD1). Functional recovery was assessed by the physiotherapist during each treatment session using the
mILAS and was reported in the electronic health record. In the intervention group, it was also reported in the app. The achievement of functional recovery on POD1 was defined as having reached a total mILAS-score of 0 on or before POD1, using a dichotomized outcome $(0=$ mILAS $=$ $0>$ POD1; 1 = mlLAS $=0 \leq$ POD1).

The independent variables measured were: Hospital Fit use (control versus the intervention group), age, sex, body mass index (BMI), type of surgery (TKA or THA), and comorbidities assessed by the American Society of Anesthesiologists (ASA) classification (ASA class $\leq 2$ versus ASA class $=3$; a higher score indicates being less fit for surgery). The medical and demographic data measured were the type of walking aid used and length of stay, with the day of surgery being defined as day 1 .

Analysis. Data analysis was performed according to the intention-to-treat principle. Missing values were not substituted; drop-outs were not replaced. Descriptive statistics were presented as means (SD) or as 95\% confidence intervals $(\mathrm{Cl})$ for continuous variables. The median and interquartile ranges (IQR) were used to present non-normally distributed data. The frequencies and percentages were used to present categorical variables. A multiple linear regression analysis was performed to determine the association between the time spent physically active per day and Hospital Fit use, corrected for potential confounding factors (age, sex, BMI, ASA class, and type of surgery). A multiple logistic regression analysis was performed additionally to determine the association between the achievement of functional recovery on POD1 and Hospital Fit use, corrected for potential confounding factors. For all statistical analyses, the level of significance was set at $P<0.05$. All statistical analyses were performed using SPSS (version 23.0.0.2; IBM Corporation, Armonk, NY).

Main results. Ninety-seven patients were recruited; after excluding 9 patients because of missing data, 88 were included for analysis, with 61 (69\%) in the control group and $27(31 \%)$ in the intervention group. A median (IQR) number of $1.00(0)$ valid measurement days ( $\geq 20 \mathrm{hr}$ wear time) was collected. Physical activity data for 84 patients (95\%) was available on POD1 ( $n=61$ control group, $n=23$ intervention group). On postoperative day 2 (POD2), 


\section{Outcomes Research in Review}

the majority of patients were discharged ( $n=61,69 \%$ ), and data for only 23 patients (26\%) were available $(n=17$ control, $n=6$ intervention). From postoperative day 3 to day 7 , data of valid measurement days were available for just 1 patient (intervention group). Due to the large reduction in valid measurement days from POD2 onward, data from these days were not included in the analysis.

Results of the multiple linear regression analysis showed that, corrected for age, patients who used Hospital Fit stood and walked an average of 28.43 minutes $(95 \% \mathrm{Cl}, 5.55-51.32)$ more on POD1 than patients who did not use Hospital Fit. Also, the model showed that an increase in age led to a decrease in the number of minutes standing and walking on POD1. The results of the multiple logistic regression analysis also showed that, corrected for ASA class, the odds of achieving functional recovery on POD1 were 3.08 times higher $(95 \% \mathrm{Cl}$, 1.148.31) for patients who used Hospital Fit compared to patients who did not use Hospital Fit. Including ASA class in the model shows that a lower ASA class increased the odds ratio for a functional recovery on POD1.

Conclusion. A smartphone app combined with an accelerometer demonstrates the potential to enhance patients' physical activity levels and functional recovery during hospitalization following joint replacement surgery.

\section{Commentary}

Although the beneficial effects of physical activity during hospitalization after surgery are well documented, patients continue to spend between $92 \%$ and $96 \%$ of their time lying or sitting..$^{1-3}$ Therefore, strategies aimed at increasing the amount of time spent standing and walking are needed. Postoperative physiotherapy aims to enhance physical activity levels and functional recovery of activities of daily living, which are essential to function independently at home. ${ }^{4-7}$ Physiotherapists may be able to advise patients more effectively on their physical activity behavior if continuous physical activity monitoring with real-time feedback is implemented in standard care. Although mobile health (mHealth) tools are being used to monitor physical activity in support of outpatient physiotherapy within the orthopedic rehabilitation pathway, ${ }^{8-10}$ there is currently no $\mathrm{mHealth}$ tool available that offers hospitalized patients and their physiotherapists essential strategies to enhance their physical activity levels and support their recovery process. In addition, because hospitalized patients frequently use walking aids and often have impaired gait, the algorithm of most available activity monitors is not validated for use in this population.

This study, therefore, is an important contribution to the literature, as it describes a preliminary evaluation of a novel mHealth tool-Hospital Fit-consisting of a smartphone application connected to an accelerometer whose algorithm has been validated to differentiate between lying/ sitting and standing/walking among hospitalized patients. Briefly, results from this study showed an increase in the time spent standing and walking, as well as higher odds of functional recovery on POD1 from the introduction of Hospital Fit. While guidelines on the recommended amount of physical activity during hospitalization do not yet exist, an average improvement of 28 minutes (39\%) standing and walking on POD1 can be considered a clinically relevant contribution to prevent the negative effects of inactivity.

This study has limitations, particularly related to the study design, which is acknowledged by the authors. The current study was a nonrandomized, quasi-experimental pilot study implemented at a single medical center, and therefore, the results have limited generalizability and more importantly, may not only be attributable to the introduction of Hospital Fit. In addition, as there was lag in patient recruitment where patients were initially selected for the control group over the course of 1 year, followed by selection of patients for the intervention group over 4 months (once Hospital Fit was developed), it is possible that awareness on the importance of physical activity during hospitalization increased among patients and health care professionals, which may have resulted in a bias in favor of the intervention group (and thus a potentially slight overestimation of results). Also, as individual functionalities of Hospital Fit were not investigated, relationships between each functionality and physical activity could not be established. As the authors indicated, future research is needed to determine the effectiveness of Hospital Fit (ie, a larger, cluster randomized controlled trial in a population of hospitalized patients with a longer length of stay). This study design would also enable investigation of the effect of individual functionalities of Hospital Fit on physical activity. 


\section{Applications for Clinical Practice}

mHealth tools have the potential to increase patient awareness, support personalized care, and stimulate self-management. This study highlights the potential for a novel mHealth tool-Hospital Fit-to improve the amount of physical activity and shorten the time to functional recovery in hospitalized patients following orthopedic surgery. Further, mHealth tools like Hospital Fit may have a greater impact when the hospital stay of a patient permits the use of the tool for a longer period of time. More broadly, continuous objective monitoring through mHealth tools may provide patients and their physiotherapists enhanced and more detailed data to support and create more personalized recovery goals and related strategies.

Katrina F. Mateo, PhD, MPH doi:10.12788/jcom.0024

\section{References}

1. Brown CJ, Roth DL, Allman RM. Validation of use of wireless monitors to measure levels of mobility during hospitalization. J Rehabil Res Dev. 2008;45:551-558.

2. Pedersen MM, Bodilsen AC, Petersen J, et al. Twenty-four-hour mobility during acute hospitalization in older medical patients. $J$ Gerontol Ser A Biol Sci Med Sci. 2013;68:331-337.

3. Evensen S, Sletvold O, Lydersen S, Taraldsen K. Physical activity among hospitalized older adults - an observational study. BMC Geriatr. 2017;17:110.

4. Engdal M, Foss OA, Taraldsen K, et al. Daily physical activity in total hip arthroplasty patients undergoing different surgical approaches: a cohort study. Am J Phys Med Rehabil. 2017;96:473-478.

5. Hoogeboom TJ, Dronkers JJ, Hulzebos EH, van Meeteren NL. Merits of exercise therapy before and after major surgery. Curr Opin Anaesthesiol. 2014;27:161-166.

6. Hoogeboom TJ, van Meeteren NL, Schank K, et al. Risk factors for delayed inpatient functional recovery after total knee arthroplasty. Biomed Res Int. 2015:2015:167643.

7. Lenssen AF, Crijns $\mathrm{YH}$, Waltje EM, et al. Efficiency of immediate postoperative inpatient physical therapy following total knee arthroplasty: an RCT. BMC Musculoskelet Disord. 2006;7:71.

8. Ramkumar PN, Haeberle HS, Ramanathan D, et al. Remote patient monitoring using mobile health for total knee arthroplasty: validation of a wearable and machine learning-based surveillance platform. J Arthroplast. 2019;34:2253-2259.

9. Ramkumar PN, Haeberle HS, Bloomfield MR, et al. Artificial Intelligence and arthroplasty at a single institution: Real-world applications of machine learning to big data, value-based care, mobile health, and remote patient monitoring. I Arthroplast. 2019;34:2204-2209.

10. Correia FD, Nogueira A, Magalhães I, et al, et al. Medium-term outcomes of digital versus conventional home-based rehabilitation after total knee arthroplasty: prospective, parallel-group feasibility study. JMIR Rehabil Assist Technol. 2019;6:e13111. 